\title{
RAC1 Involves in the Radioresistance by Mediating Epithelial-Mesenchymal Transition in Lung Cancer
}

\begin{abstract}
Shiming Tan ${ }^{1 \dagger}$, Pin $\mathrm{Yi}^{1,2 \dagger}$, Heran Wang ${ }^{1,3}$, Longzheng Xia ${ }^{1}$, Yaqian Han ${ }^{1}$, Hui Wang ${ }^{1}$, Biao Zeng ${ }^{1}$, Lu Tang ${ }^{1,2}$, Qing Pan 1,2, Yutong Tian 1,2, Shan Rao ${ }^{1}$, Linda Oyang ${ }^{1}$, Jiaxin Liang ${ }^{1}$, Jinguan Lin ${ }^{1}$, Min Su ${ }^{1}$, Yingrui Shi ${ }^{1}$, Qianjin Liao ${ }^{1 *}$ and Yujuan Zhou ${ }^{1 *}$

${ }^{1}$ Hunan Key Laboratory of Translational Radiation Oncology, Hunan Cancer Hospital and The Affiliated Cancer Hospital of Xiangya School of Medicine, Central South University, Changsha, China, ${ }^{2}$ Hunan Cancer Hospital, University of South China, Hengyang, China, ${ }^{3}$ Hepatology Unit, Department of Infectious Disease, Nanfang Hospital, Southern Medical University, Guangzhou, China
\end{abstract}

\section{OPEN ACCESS}

Edited by:

Boris Zhivotovsky,

Karolinska Institutet (KI), Sweden

Reviewed by:

David Sarrio,

Center for Biomedical Research in Cancer Network (CIBERONC), Spain

Frederique Gaits-lacovoni,

Institut National de la Santé et de la

Recherche Médicale

(INSERM), France

*Correspondence:

Qianjin Liao

march-on@126.com

Yujuan Zhou

yujany_zhou@163.com

†These authors have contributed equally to this work

Specialty section

This article was submitted to Molecular and Cellular Oncology, a section of the journal

Frontiers in Oncology

Received: 06 January 2020 Accepted: 07 April 2020

Published: 28 April 2020

Citation:

Tan S, Yi P, Wang H, Xia L, Han Y,

Wang H, Zeng B, Tang L, Pan Q. Tian Y, Rao S, Oyang L, Liang J, Lin J, Su M, Shi Y, Liao Q and Zhou Y (2020) RAC1 Involves in the Radioresistance by Mediating Epithelial-Mesenchymal

Transition in Lung Cancer.

Front. Oncol. 10:649.

doi: 10.3389/fonc.2020.00649
Radiation therapy is a common and acceptable approach for lung cancer. Although the benefit of ionizing radiation (IR) is well-established, cancer cells can still survive via pro-survival and metastasis signaling pathways. Ras related C3 botulinum toxin substrate1 (RAC1), a member of Rho family GTPases, plays important roles in cell migration and survival. In the present study, we investigated the effects of RAC1 on the survival of lung cancer cells treated with irradiation. The results showed RAC1 is overexpressed in lung cancer cells and promoted cell proliferation and survival. Furthermore, IR induced RAC1 expression and activity via the activation of PI3K/AKT signaling pathway, and then enhancing cell proliferation, survival, migration and metastasis and increasing levels of epithelial-to-mesenchymal transition (EMT) markers, which facilitated the cell survival and invasive phenotypes. In addition, overexpression of RAC1 attenuated the efficacy of irradiation, while inhibition of RAC1 enhanced sensitivity of irradiation in xenograft tumors in vivo. Collectively, we further found that RAC1 enhanced radioresistance by promoting EMT via targeting the PAK1-LIMK1-Cofilins signaling in lung cancer. Our finding provides the evidences to explore RAC1 as a therapeutic target for radioresistant lung cancer cells.

Keywords: RAC1, lung cancer, radioresistance, epithelial-to-mesenchymal transition, metastasis

\section{INTRODUCTION}

Lung cancer is the most common cancer and one of the leading causes of death worldwide (1). The majority of lung cancer is NSCLC (non-small cell lung cancer) includes squamous cell carcinoma, adenocarcinoma, and large cell carcinoma (2). Medical treatments to cure NSCLC are mainly surgery, radiation therapy, and chemotherapy (3). Radiation therapy combined with chemotherapy can produce a cure only in a small number of NSCLC patients because NSCLC is comparatively less sensitive to chemotherapy or radiation $(4,5)$. Acquired resistance results in local tumor recurrence or failure of radiotherapy $(6,7)$. Increasing evidence has demonstrated that epithelial-to-mesenchymal transition (EMT) mediated tumor metastasis is closely associated with radiation resistance $(8,9)$. The biological process during EMT endows epithelial cells lose their apical-basal polarity and acquire mesenchymal cell traits $(10,11)$, which is characterized by the loss of epithelial morphology and the acquisition of mesenchymal morphology, of which the epithelial markers includes E-cadherin, Desmoplakin, Occludins, Claudins, and ZO-1) and 
mesenchymalmarkers includs $\mathrm{N}$-cadherin, vimentin, and FSP1. Moreover, the activation of Wnt, TGF- $\beta$, and NF- $\kappa$ B signaling pathway are validated in this process (12-14). Many radioresistant cancer cells demonstrate EMT is considered to link to adaptation to hypoxia (15), enhanced DNA repair capacity $(10,15,16)$, activated molecular events, and signaling pathways (17).

Ionizing radiation (IR) therapy is routinely used for lung cancer treatment $(9,18)$. IR could induce DNA damage in cancer cells and subsequently lead to cell death. However, the survival of cancer cells with radioresistance to IR remained a major problem in radiotherapy (19). Thus, it is important to improve understanding of the mechanisms that cancer cells bear IRinduced cytotoxicity. It is well-established that several pathway are cancer cells activated to promote DNA repair and cell survival in response to IR (20), such as cell cycle checkpoint activation, DNA repair pathway, ATM/ATR, PI3K/AKT, and MEK/ERK activation, which promotes survival of cancer cells through up-regulation of anti-apoptotic factors and down-regulation of pro-apoptotic factors $(12,21-24)$. In addition, The changes in the tumor microenvironment (TME) induced by IR promote tumor invasion and metastasis, which is accompanied with EMT occurrence (25-28). Therefore, it is necessary to explore the relationship of IR and EMT and uncover the potential mechanisms of radioresistance.

Ras-related C3 botulinum toxin substrate 1 (RAC1), as a member of the Rho family of small guanosine triphosphatases (GTPases), has been shown to play a critical role in cytoskeleton reorganization, cell migration and cell survival (29). RAC1 has been found to be overexpressed in various tumors, and is closely associated with tumor migration, invasion and poor prognosis of carcinomas (30). Down-regulated of RAC1 expression or loss of its function significantly suppressed cancer cell proliferation and metastasis (31). Recent study found RAC1 pathway was activated in the regulation of breast cancer cell response to IR is to induce $\mathrm{G}_{2} / \mathrm{M}$ checkpoint activation (32). In contrast, inhibition of RAC1 GTPase sensitizes to $\gamma$-irradiation in pancreatic cancer cells (33). However, the role of RAC1 in lung cancer radiotherapy is poorly understood. In this study, the function of RAC1 in human lung cancer cells and its role in response to IR were investigated.

\section{MATERIALS AND METHODS}

\section{Cell Culture and Treatment}

A549, PC9, H1299, H460, and HBE cells were maintained in RPMI-1640 medium supplemented with 10\% FBS and 100 $\mathrm{U} / \mathrm{ml}$ penicillin-streptomycin solution (Bioind, Israel) in 5\% $\mathrm{CO}_{2}$ incubator. CMV-RAC1, CMV-sh-RAC1 and their controls plasmids were purchased from Shanghai Genechem Co., LTD. A549 and PC9 Cells transfected using Lipofectamine ${ }^{\mathrm{TM}} 3000$ (Invitrogen, Waltham City, Massachusetts, USA) with vector, RAC1, sh-control and sh-RAC1 plasmids followed by $500 \mu \mathrm{g} / \mathrm{ml}$ G418 screening were pooled collected and positive clones were selected and identified for stably expressing RAC1 or silencing RAC1. For experiments involving IR exposure, growing cells with serum-free culture medium after indicated dose of $2 \mathrm{~Gy} / \mathrm{min}$ were continuously incubated with $10 \%$ FBS at $37^{\circ} \mathrm{C}$ for $48 \mathrm{~h}$ prior to analysis.

\section{Antibodies}

Anti-E-cadherin (ab15148), anti-Vimentin (ab92547), anti$\mathrm{N}$-cadherin (ab98952), anti-RAC1 (ab33186), anti-PAK-1 (ab40582), anti-phospho-PAK-1 (ab40795), anti phosphoLIMK1 (ab31341), anti-Ki67 (ab15580), and anti-Caspase-3 (ab13847) was from Abcam (London, UK). Anti-LIMK1 (G22412E9) was from Abnova (Taipei, China). Anti-Cofilin1 (\#5175) and anti-phospho-Cofilin1 (\#3311) were from Cell Signaling Technology (Danvers, MA, USA); anti-GAPDH (60004-1-Ig) was from Proteintech (Chicago, Illinois, USA). HRP-labeled goat anti-rabbit IgG and HRP-labeled goat anti-mouse IgG were from Beyotime (Shanghai, China).

\section{Establishment of Stably Rac1-Overexpression/Interfering Cell Lines} PCMV-Racl and PCMV sh-RAC1 interfering plasmids were used to obtain A549 and PC9 stable expressing RAC1 or stable down-regulated RAC1 according to the protocol in our previous study (34). The target sequences of scramble and shRAC1 were $5^{\prime}$-TTCTCCGAACGTGTCACGT-3' and $5^{\prime}$ TGCAGTAGATGATGAAAGAAA-3', respectively.

\section{Cell Proliferation Assay}

A549 and PC9 cells numbers was measured as previously described (35). Briefly, cells were then seeded at 96-well microplates at a density of $3 \times 10^{3}$ cells/well. CCK- 8 assay was performed to assess the viability according to the manufacturer's protocol. The experiment was performed in triplicate.

\section{Clone Formation Assay}

Clone formation assay was performed as described previously (36). Briefly, 300 cells were seed at 6-well microplates and incubated for 10-14 days until colonies formed. For IR-induced experiment, different numbers of cells were exposed to IR at an indicated dose of $0,2,4,6,8 \mathrm{~Gy}$ (37) and the colonies were visualized by crystal violet staining and quantified using Image J software.

\section{Wound-Healing Assay}

Cells were seeded into 6-well plates and cultured up to $80 \%$ cell density (Corning, Corning City, State of New York, USA). The artificial "wound" was scratched by a $10 \mu \mathrm{l}$ pipette tip and culture with serum free culture medium. Measure the gap size followed by photographing using an inverted microscope.

\section{Transwell Migration and Invasion Assays}

Transwell migration and invasion assays were performed as described previously (36). The cells were stained with crystal violet and counted under a microscope (Olympus Corp, Tokyo, Japan). The results were statistically analyzed.

\section{Western Blot Analysis}

Western blotting was performed as described previously (36). The blots were visualized using Immobilon Western Chemiluminescent HRP Substrate (EMD Millipore). Images of 
the western blotting products were captured and analyzed using Quantity One V4.31 (Bio-Rad Laboratories, Inc.).

\section{RAC1 Pull-Down Assays}

Racl pulldown assay was performed using commercial kit (\#8815, CST) as described recently (36). RAC1-GTP was detected by western blotting using antibodies specific for RAC1 $(1: 100$, CST).

\section{Quantitative Real-Time PCR (qRT-PCR)}

Total RNA was extracted from the cells with TRIzol (Invitrogen; Thermo Fisher Scientific, Inc.) and total RNA $(1 \mu \mathrm{g})$ was used to synthesize cDNA using (cDNA synthesis kit Thermo Fisher Scientific, Inc.). The reaction system was applied according to the manufacturer's protocol: $42^{\circ} \mathrm{C}$ for $15 \mathrm{~min}$ and $95^{\circ} \mathrm{C}$ for $3 \mathrm{~min}$. The PCR primers (GAPDH, forward 5'-AGCGAGCATCCC CCAAAGTT-3', reverse 5'-GGGCACGAAGGCTCATCATT-3'; RAC1, forward 5'-CCCTATCCTATCCGCAAACA-3', reverse $5^{\prime}$-CGCACCTCAGGATACCACTT-3'; and E-cadherin, forward $5^{\prime}$-ACTGGGACGACGACATGGAAAAG- ${ }^{\prime}$ and reverse $5^{\prime}$-TAG ATGGGGACATTGTGGGT- $3^{\prime}$ ) were designed and validated by Sangon Biotech Co., Ltd. (Shanghai, China). qPCR was performed at $95^{\circ} \mathrm{C}$ for $5 \mathrm{~min}$, followed by 40 cycles of amplification at $95^{\circ} \mathrm{C}$ for $10 \mathrm{~s}$ and $60^{\circ} \mathrm{C}$ for $30 \mathrm{~s}$ on an Applied Biosystems 7500 Real-Time PCR system (Applied Biosystems; Thermo Fisher Scientific, Inc.). The fluorescent signals were collected during the extension phase, quantification cycle (Cq) values of the sample were calculated, and transcript levels were analyzed using the $2-\Delta \Delta \mathrm{Cq}$ method. The results were normalized to GAPDH.

\section{Immunohistochemistry (IHC)}

Immunohistochemistry were performed as previously described (36). The specimens were paraffin embedded, and the paraffin sections were subject to antigen retrieval by microwave irradiation in citrate buffer, then treated with $0.3 \% \mathrm{H}_{2} \mathrm{O}_{2}$, PBS $(\mathrm{pH} 7.35-7.45)$ and further blocked in $3 \%$ BSA/PBS. After hydration, the paraffin sections were executed according to the immunohistochemical kit (Cwbiotech Company, Beijing, China) protocols. Anti-Rac1 (1: 150), anti-Ki67 (1:500), and anti-caspase3 (1:150) were used as the primary antibodies. The samples were scored according to the staining intensity and number of positive cells as described previously (38). (1) staining intensity, no observed cell staining was scored as 0 , cells with weak staining as 1 , cells with moderate staining as 2 , cells with moderate staining as 3; (2) number of positive cells, no positive cells were scored as $0,<25 \%$ of positive cells as 1 , between 25 and $50 \%$ positive cells as 2, positive cells over $50 \%$ as 3 . Next, the score was obtained by multiplying the intensity and reactivity rates. Scores of $<4$ suggested low expressions, and the remainder were classified as high expression. The immunostaining results were confirmed independently by two pathologists in a blinded manner.

\section{Animal Experiments}

Animal experiment was also performed as previously described (36). Briefly, a total of $5 \times 10^{6}$ cells were subcutaneously injected into the nude mice. The tumor sizes were measured with calipers every other day. Tumor size was estimated by the modified ellipsoid formula: $1 / 2$ (length $\times$ width $^{2}$ ). When the tumor size reached up to $\sim 120 \mathrm{~mm}^{3}$, tumors were irradiated with a dose of $15 \mathrm{~Gy}$ ( $5 \mathrm{~Gy} \times 3$ fractions). Body weights and tumor volumes were measured every other day. Tumor volumes were calculated by measuring the length $[\mathrm{L}]$ and width $[\mathrm{W}]$ of tumors using calipers. The formula tumor volume $=\left(\mathrm{L} \times \mathrm{W}^{2}\right) / 2$ was used to calculate the tumor volume. This experiment was conducted in accordance with the National Institutes of Health Guide for the Care and Use of Laboratory Animals and approved by the Institutional Animal Care and Use Committee of the Hunan Cancer Hospital and The Affiliated Cancer Hospital of Xiangya School of Medicine, Central South University (Changsha, China).

\section{Statistical Analysis}

All statistical analyses were conducted using PRISM GraphPad 7.0. Statistical analyses were performed by means of the Student's $t$-test and the Mann-Whitney $U$ test. Data are presented as the mean \pm standard deviation. $P<0.05$ was considered to indicate a statistically significant difference.

\section{RESULTS}

\section{RAC1 Regulates Cell Proliferation in Lung Cancer Cells in vivo and in vitro}

Overexpression of RAC1 has been detected in the great majority of lung cancers (39). Our previous study have been elucidated that RAC1 expression is correlated with the expression of EMT markers in non-small cell lung cancer (NSCLC) (38). We first analyzed RAC1 mRNA and protein expression in normal lung cell (HBE) and four NSCLC cell lines (H460, H1299, A549, and PC9). The results showed that RAC1 expression was elevated in four NSCLC cell lines relative to normal lung HBE epithelial cells (Figures S1A,B). We then examined the biological function of RAC1 in lung cancer cells. PC9 and A549 cells with stable RAC1 or sh-RAC1 transfection had moderate RAC1 expression levels (Figure 1A and Figure S1C). The colony formation assays showed that ectopic overexpression of RAC1 promoted growth of both A549 and PC9 cells compared to empty vector control (Figure 1B). In contrast, inhibition of RAC1 decreased the ability of colony formation (Figure 1B). Furthermore, CCK-8 assays demonstrated that overexpression of RAC1 promoted cell proliferation, but silencing of RAC1 expression inhibited cell proliferation in both A549 and PC9 cells (Figure 1C). Tumor increased at a much rapid rate in nude mice in the RAC1 compared with the control group $(P<0.05)$ (Figures 1D,E), while tumor weight was significantly larger in the RAC1 group (Figure 1F). On the other hand, tumor increased at a lower rate in nude mice in the shRAC1 compared with sh-control group, and tumor weight was smaller in the sh-RAC1 group (Figures 1D,E). These results suggest that RAC1 promotes proliferation of lung cancer cells. 


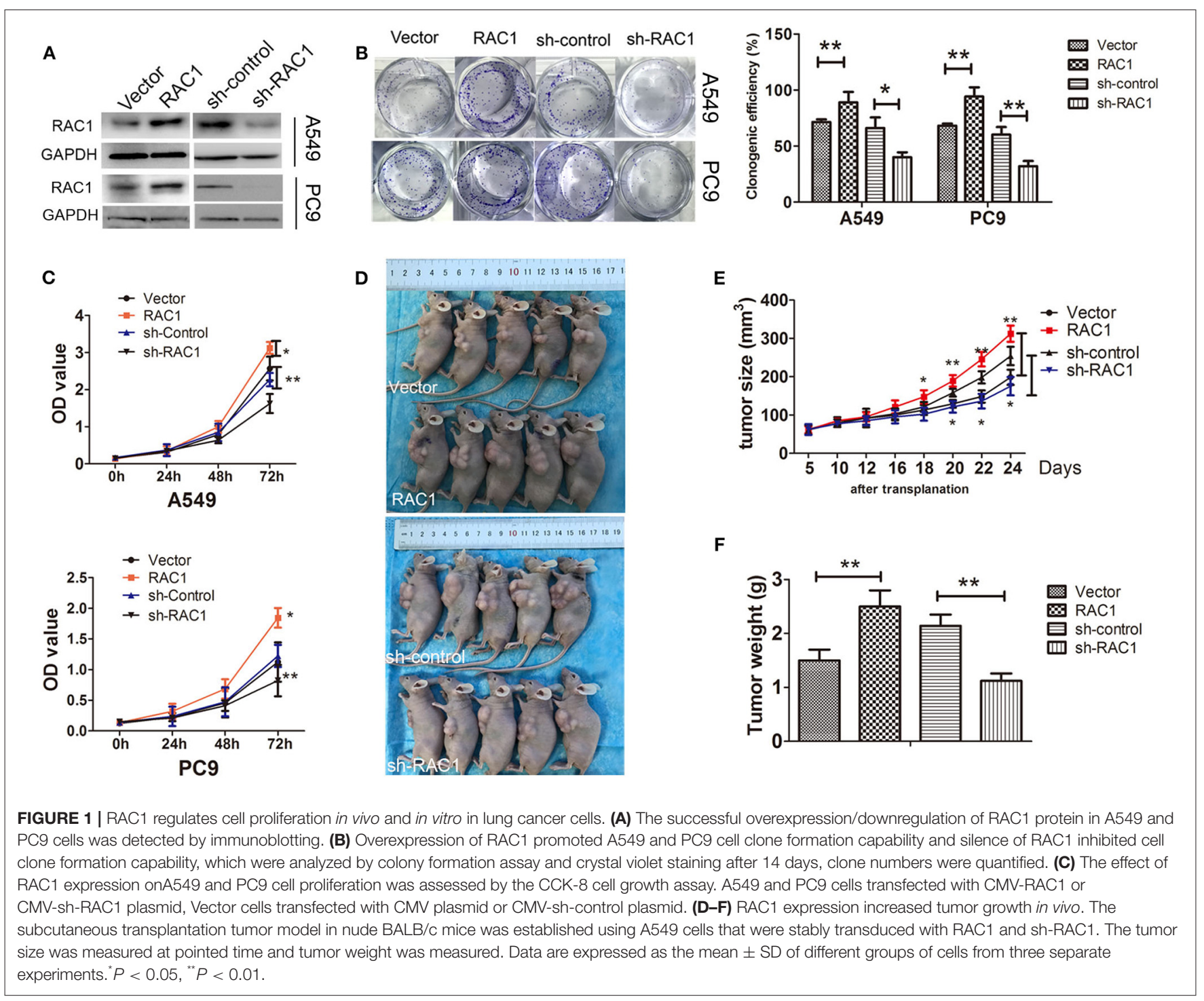

\section{IR Induces RAC1 Expression and EMT in Lung Cancer Cells}

Our previous study demonstrated that RAC1 is closely related to radioresistance in patient samples with lung cancer (38). Herein, we found the mRNA expression levels of RAC1 were up-regulated with the increased dose of X-rays $(2,4,6$, and $8 \mathrm{~Gy}$ ) up to a maximum level at $8 \mathrm{~Gy}$ (Figure 2A). The protein expression of RAC1 showed a similar tendency, in which the protein expression of RAC1 was significantly upregulated at 4, 6, and $8 \mathrm{~Gy}$ (Figure $2 \mathrm{~B}$ ). In addition, as shown in Figure 2C, the results of GST-pull down assays showed Rac1 expression and activity was significantly increased after 6 Gy dose of IR in lung cancer cells, suggesting that IR could promote the Racl expression and activity. A question is how IR induces Racl expression. According to the report that IR could activate the PI3K/AKT signaling pathway, so we next detected the expression of the effector proteins of the PI3K/AKT signaling pathway after IR, such as PI3K, p$\mathrm{AKT}$, and AKT. As shown in Figure 2D, the immunoblotting results showed that the $\mathrm{PI} 3 \mathrm{~K}$ and $\mathrm{p}$-AKT were significantly up-regulated with 6 Gy dose of IR in A549 and PC9 cells. It suggested that IR might induce the activation of PI3K/AKT signaling pathway to promote the Racl expression. To investigate whether or not the activation of PI3K/AKT signaling pathway could increase the expression of Racl, we use the class I PI3K inhibitors, LY294002, to treat the A549 and PC9 cells with 6 Gy dose of IR. The western blot results showed that IR could significantly increase the PI3K, p-AKT, AKT, and RAC1, whereas the LY294002 reversed this effect in both A549 and PC9 cells (Figure 2E). It indicated that Rac1 was the target of the PI3K/AKT signaling pathway, the same as the previous study (36). These results indicate that IR increases the expression and activity of Racl via activating the PI3K/AKT signaling pathway. 


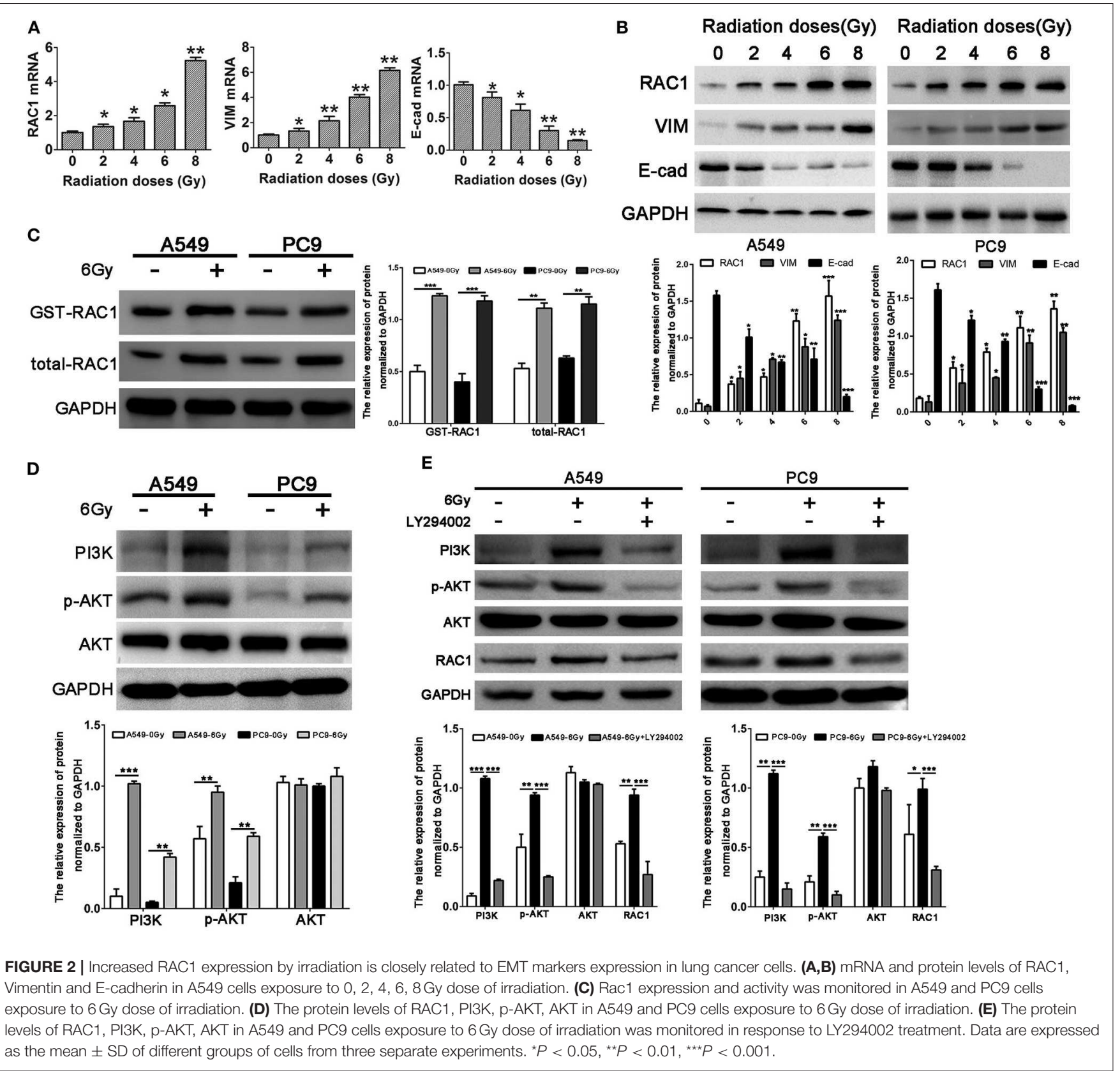

\section{RAC1 Promotes Radioresistance, Invasion and Migration in Lung Cancer Cells}

Previous study suggested that up-regulated RAC1 by IR may promote carcinogensis of lung cancer. To assess the effects of RAC1 on the resistance of lung cancer cells to IR, cells were stably transfected with RAC1 (Figure 1A), and then exposed to IR before the survival fraction was analyzed using a colony-forming assay and cell proliferation using CCK- 8 assay. The results demonstrated that RAC1 increased survival capacity of A549 and PC9 cells after IR at 0, 2, 4, 6, and 8Gy (Figure 3A). In contrast, silencing of RAC1 by sh-RAC1 contributed to the sensitivity of lung cancer cells to IR; cells transfected with sh-RAC1 also displayed decreased survival capacity by evaluating survival fraction using a colonyformation assay (Figure 3A). Furthermore, a decrease of cell proliferation was found in A549 and PC9 cells with sh-RAC1 (Figure 3B).

We then investigated whether effects of IR on invasion and migration were mediated by RAC1 expression in lung cancer cells. IR promoted A549 and PC9 cells invasion and migration, while down-regulation of RAC1 reversed the effects of IR (Figure S2). In addition, wound healing assay, invasion and migration assay showed that RAC1 

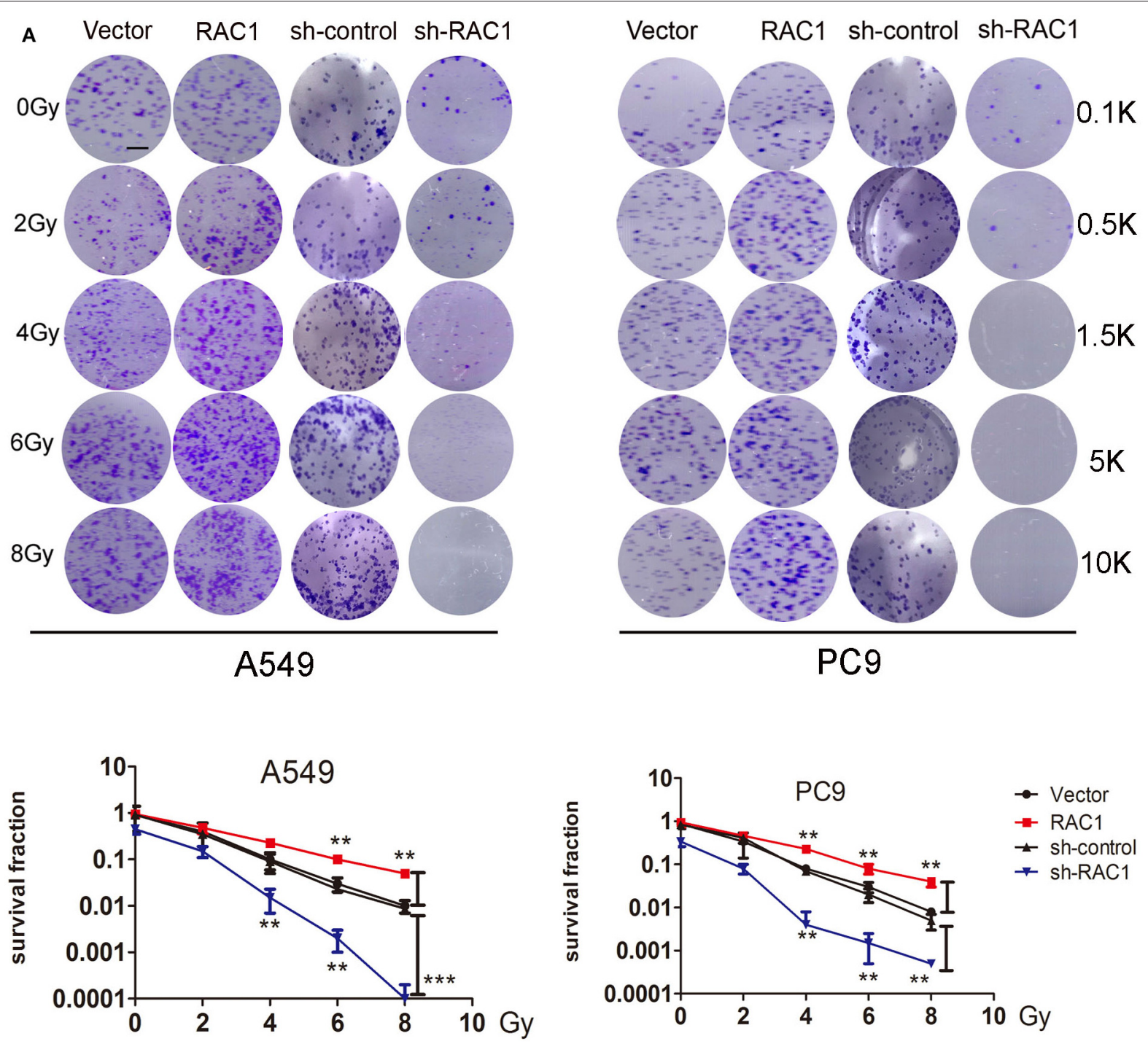

B
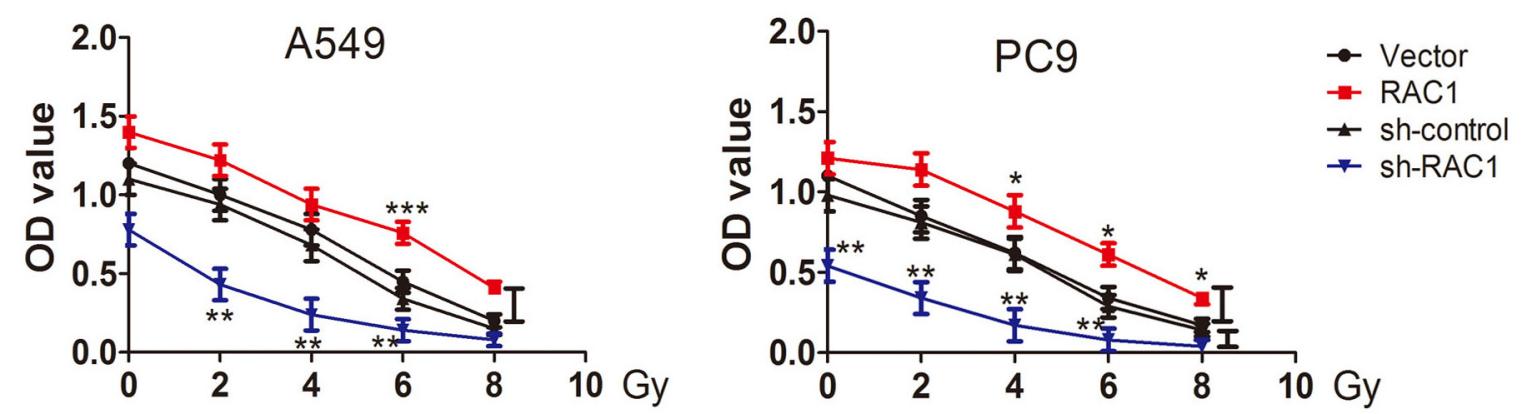

FIGURE 3 | RAC1 inhibition increased the radio-sensitivity for lung cancer cells. (A) RAC1-overexpressing A549 and PC9 cells exposure to 0, 2, 4, 6, 8 Gy dose of irradiation promoted cellular radioresistance, and RAC1 silenced A549 and PC9 cells exposure to 0, 2, 4, 6, 8 Gy dose of irradiation attenuated cellular radioresistance under as determined by the clonogenic assay. (Down panel) Dose-survival curve derived from the results of clonogenic assay for A549 and PC9 cells.

$\mathrm{SF}=$ colonies/(inoculation cells $\times$ survival rate). SF represents the sensitivity of cells to RAC1 expression exposed to IR. These numbers (0.1, $0.5,1.5,5$, and $10 \mathrm{k})$ represent the number of seeded cells (100, 500, 1,500, 5,000, and 10,000). (B) Cell proliferation ability of A549 and PC9 cells within indicated group were detected by CCK-8 assay. Data are expressed as the mean \pm SD of different groups of cells from three separate experiments. ${ }^{\star} P<0.05$, ${ }^{\star \star} P<0.01,{ }^{\star \star \star} P<0.001$. 

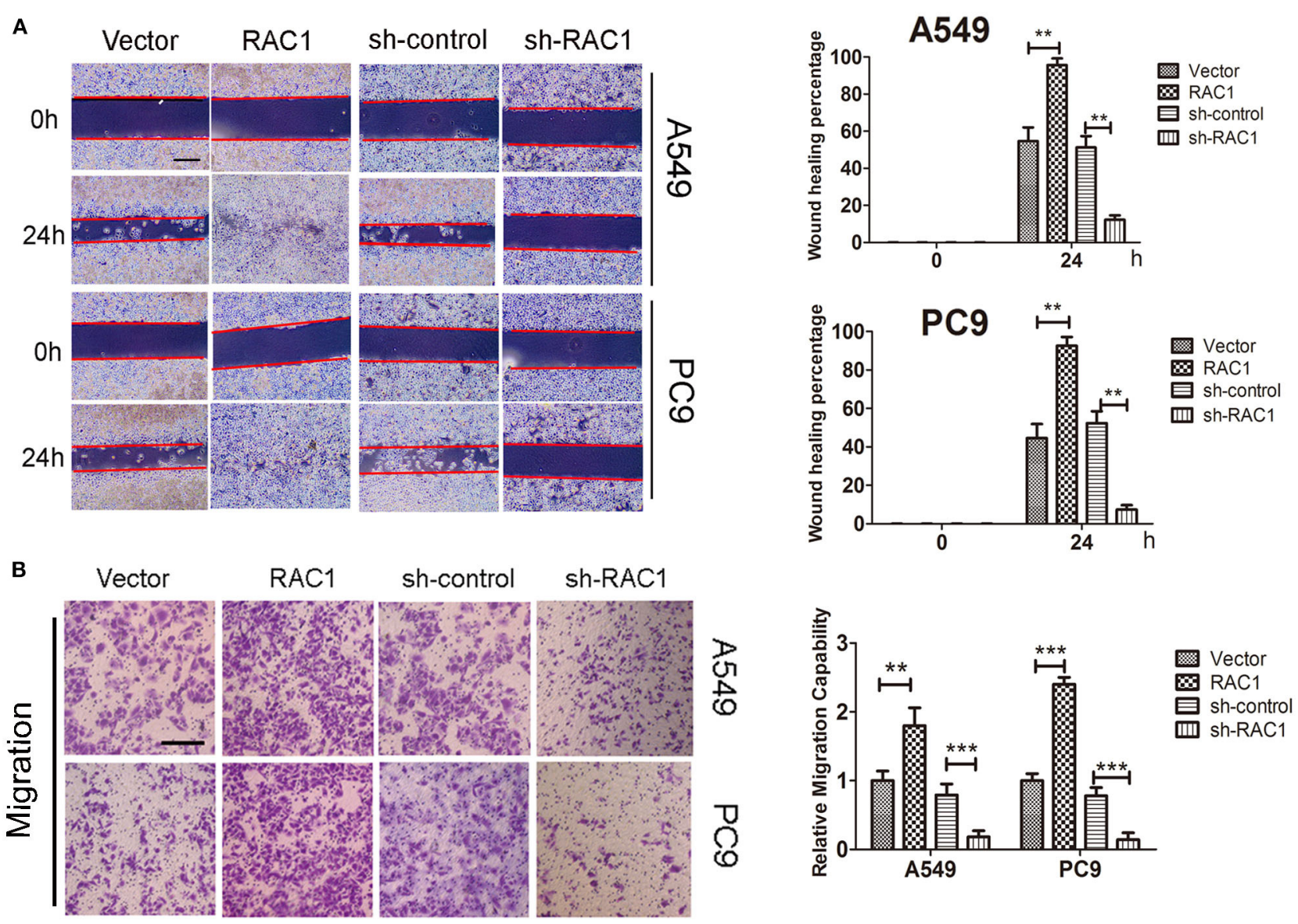

C Vector

RAC1

sh-control

sh-RAC1
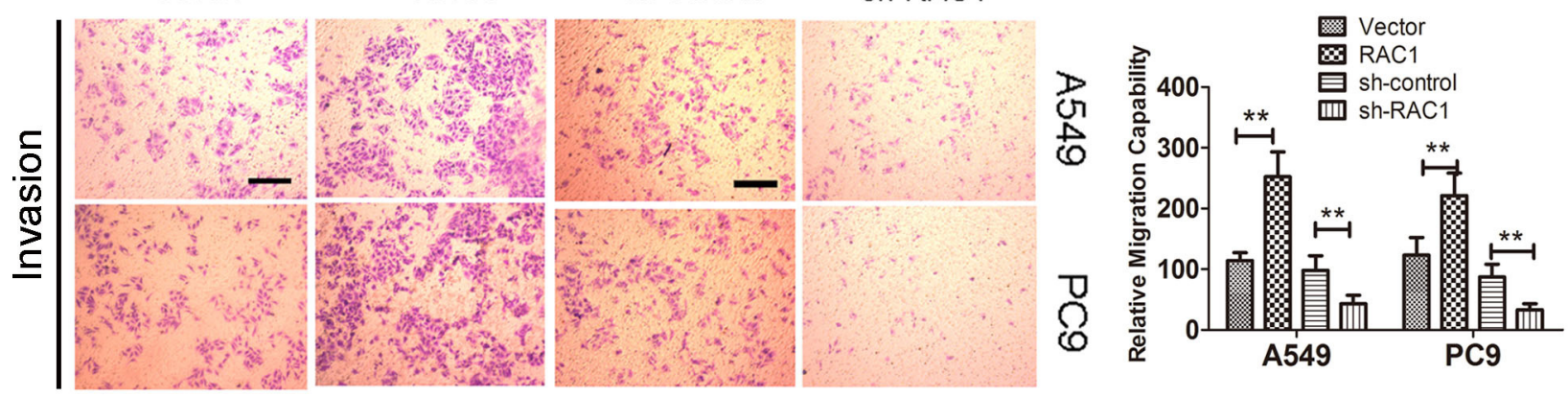

FIGURE 4 | The effects of RAC1 expression on irradiation-induced migration and invasion in vitro. A549 and PC9 cells were exposed to 6 Gy doses of IR in the presence or absence of RAC1 expression. (A) The influence of RAC1 expression on lung cancer cell migration was determined by wound healing assay in A549 and PC9 cells. Scale bar is $100 \mu \mathrm{m}$. (B) The effects of RAC1 expression on cell migration detected by Transwell assay, scale bar is $50 \mu \mathrm{m}$. (C) The effects of RAC1 expression on cell invasion detected by Transwell assay, scale bar is $50 \mu \mathrm{m}$. Data are expressed as the mean \pm SD of different groups of cells from three separate experiments. ${ }^{\star \star} P<0.01,{ }^{\star \star \star} P<0.001$.

promoted cells migration after IR at $6 \mathrm{~Gy}$ exposures in A549 and PC9 cells (Figures 4A-C). These results indicate that IR induces the radioresistance and promotes the invasion and migration of lung cancer cells mediated by RAC1 levels.

\section{IR Induces EMT Phenotypes via Regulating RAC1 Signaling Pathway}

Epithelial-mesenchymal transition (EMT) involves dramatic reorganization of the cytoskeleton, which is a crucial initiating step in tumor invasion and metastasis. IR induces EMT via 
down-regulating expression of epithelial marker (E-cadherin) and increasing mesenchymal marker expression (vimentin). Herein, we confirmed that IR induced EMT via down-regulating expression of E-cadherin and increasing vimentin expression in A549 and PC9 cells with both the knockdown and overexpression of RAC1 on EMT in IR-treated and untreated cells (Figure 5). Subsequently, further to verify whether RAC1 signaling pathway was involved in the IR-triggered EMT in lung cancer cells, we detected the expression of RAC1 signaling pathway molecules in both the knockdown and overexpression of RAC1 on EMT in IR -treated and untreated cells. As exhibited in Figure 5, RAC1 overexpression led to the up-regulation of GST-RAC1, RAC1, PAK1, p-PAK1, LIMK1, p-LIMK1, Cofilin, and p-Cofilin in A549 and PC9 cells, while the opposite pattern of these genes was found in the A549 and PC9 cells after Rac1 knockdown. IR significantly promoted the expression of GST-RAC1, RAC1, PAK1, p-PAK1, LIMK1, p-LIMK1, Cofilin, and p-Cofilin in the cells treated with IR. The effect of IR on these EMT related markers and molecular markers of RAC1 signaling pathway could be partly augmented by RAC1 overexpression and reversed by RAC1 knockdown (Figure 5). Therefore, these data suggest that RAC1 signaling pathway is involved in the course of IR-induced EMT, and RAC1 is a potential target molecule for the inhibitory effects of IR on lung cancer cell EMT.

\section{RAC1 Regulates Radioresistance of Transplanted Tumors in Nude Mice}

We next investigated whether RAC1 could affect NSCLC cellular response to IR in vivo. RAC1-overexpressing A549 cells or shRAC1 cells and their control cells were inoculated into 4-6 weeks of female nude mice. When tumors reached a size of at least $120 \mathrm{~mm}^{3}$, the xenografts were irradiated at a dose of $15 \mathrm{~Gy}(5 \mathrm{~Gy} \times 3$ fractions). Consistent with the in vitro results, RAC1 significantly enhanced tumor xenograft growth treated with IR (Figures 6A,B). In contrast, sh-RAC1 group showed a decreased size of tumors responding to IR, but sh-RAC1 significantly inhibited the tumor xenograft growth treated with IR (Figures 6A,B). The expressions of RAC1, Ki67 , and activated caspase- 3 were detected in the xenograft tumor tissues of A549 cells in mice (Figure 6C). Increased Ki67 and RAC1 expression and decreased activated caspase-3 levels were observed in the RAC1 over-expression group compared to the Vector group, while this effect was significantly reversed in group with IR treatment (Figure 6C). In addition, the reduced the levels of Ki67 and RAC1, and raised activated caspase-3 were observed in sh-RAC1 group, but IR could enhance the effect (Figure 6C). These results in vivo and in vitro suggest RAC1 regulates radio-resistance in lung cancer.

\section{DISCUSSION}

RAC1 is constitutively activated in a great majority of lung cancer and contributes critically to the development and progression of lung cancer via EMT (40). In the present study, we observed a striking up-regulation of RAC1 level in NSCLC cells. The RAC1 signaling pathway is required for transformation mediated by the Ras oncogene (41). The RAC1 pathway promotes transformation, protects against apoptosis, and promotes motility and invasion (42-44). It has been reported that Racl is the target molecule of the PI3K/AKT pathway. When the PI3K/AKT pathway was activated, the expression and biological activity of Racl were significantly up-regulated (36). Meanwhile, RAC1b expression is positively associated with increased growth and chemo-resistance via enhancing NF- $\kappa \mathrm{B}$ activity and then activating NF- $\kappa \mathrm{B}$ signaling in colorectal cancer (45). In this report, we provide evidence that RAC1 promoted cell proliferation and colony formation in lung cancer cells; silencing of RAC1 expression inhibited pro-survival signal of lung cancer in vivo and in vitro. These evidences strongly indicate that RAC1 contributes to lung cancer progression and maybe a useful prognostic biomarker of lung cancer.

IR plays a crucial role in cancer treatment; however, radio-resistance leads to distant metastases in patients with radiotherapy (46). Accumulated evidence has demonstrated the important role of EMT in metastasis (47). In this study, we found that IR time-dependently induced EMT in A549 and PC9 cells as indicated by the expression of EMT marker proteins. In lung cancer cells, IR can induce the EMT via activating TGF- $\beta$ signaling pathway. In this report, we found that IR could induce the up-regulation of RAC1 expression and activity via activating the PI3K/AKT signaling pathway $(36,48-50)$. Interestingly, IRinduced EMT is regulated by RAC1, a member of the Rho family of small guanosine triphosphatases (GTPases) that has been shown to play a critical role in cytoskeleton reorganization, cell migration and cell survival $(51,52)$. Thus, we raised the possibility that RAC1 might be an important regulator in the process of IR-induced EMT in lung cancer.

Recently, it has been identified that the RAC1 signaling pathway is an important regulator of the response of breast and pancreatic cancer cells to $\operatorname{IR}(32,33)$. E.g., RAC1 is activated by IR and the inhibition of RAC1 abrogates G2 checkpoint activation and cell survival following IR in breast cancer cells $(32,53)$. Biochemical analyses indicate that RAC1 inhibition alone could reduce phosphorylation of ERK1/2 and I $\mathrm{B} \alpha$, as well as levels of anti-apoptotic proteins Bcl-xL and Mcl-1L in the non-irradiated HFR-selected cells (32). The similar role played by RAC1 was observed in pancreatic cancer cells (32). Pancreatic cancer cells are notoriously resistant to the toxicity of radiation therapy $(54,55)$. In this article, we uncovered inhibition of RAC1 in lung cancer cells is sufficient to abrogate the IR-induced and RAC1-mediated tumor migration and invasion, as evidenced by cell proliferation, colony formation, and Transwell assay. In addition, activation of RAC1 accelerates this process induced by IR. These findings suggest RAC1 contributes to the regulation of radioresistance in lung cancer cells. The inhibition of RAC1 also abrogates the IR-induced mesenchymal markers vimetin and Snail, which plays an important role in EMT. These results reveal that RAC1 protect lung cancer cells from the cytotoxic effects in response to IR. These data raises the possibility that the intrinsic radioresistance of lung cancer cells might be a consequence of the constitutive activation of the RACl pathway in this disease. 


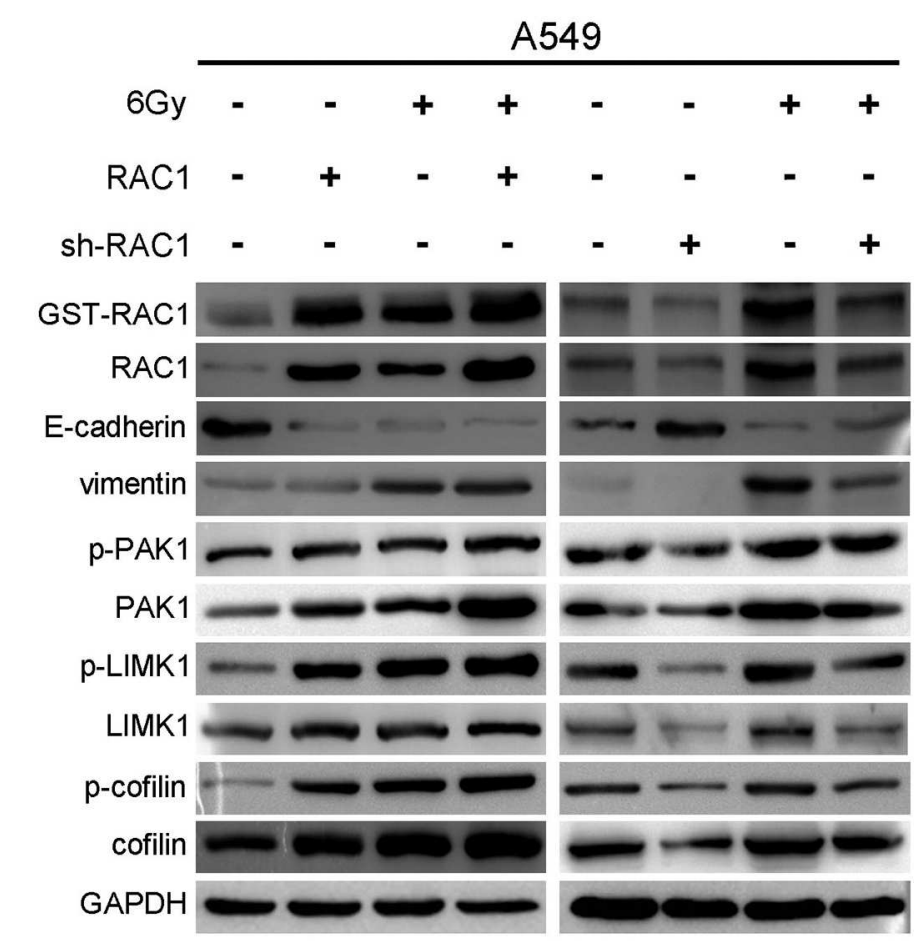

A549

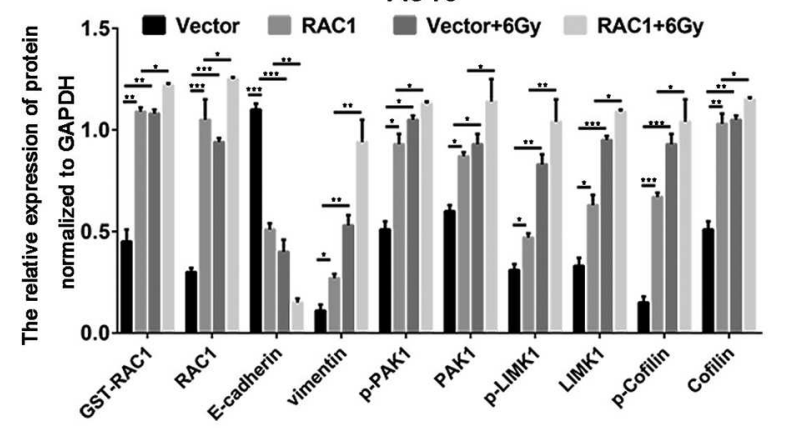

PC9

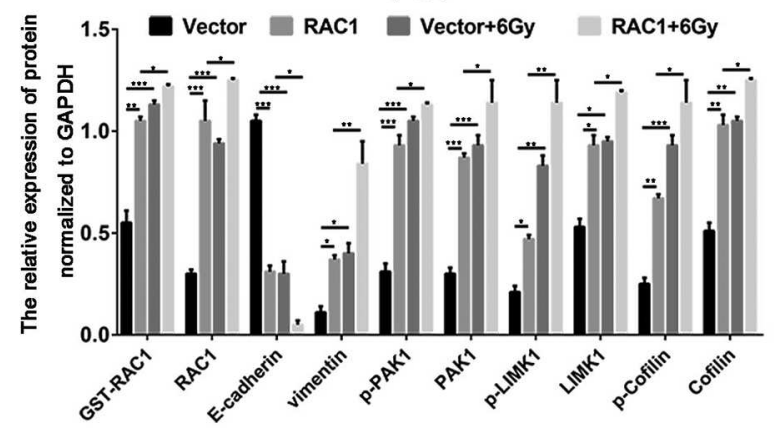

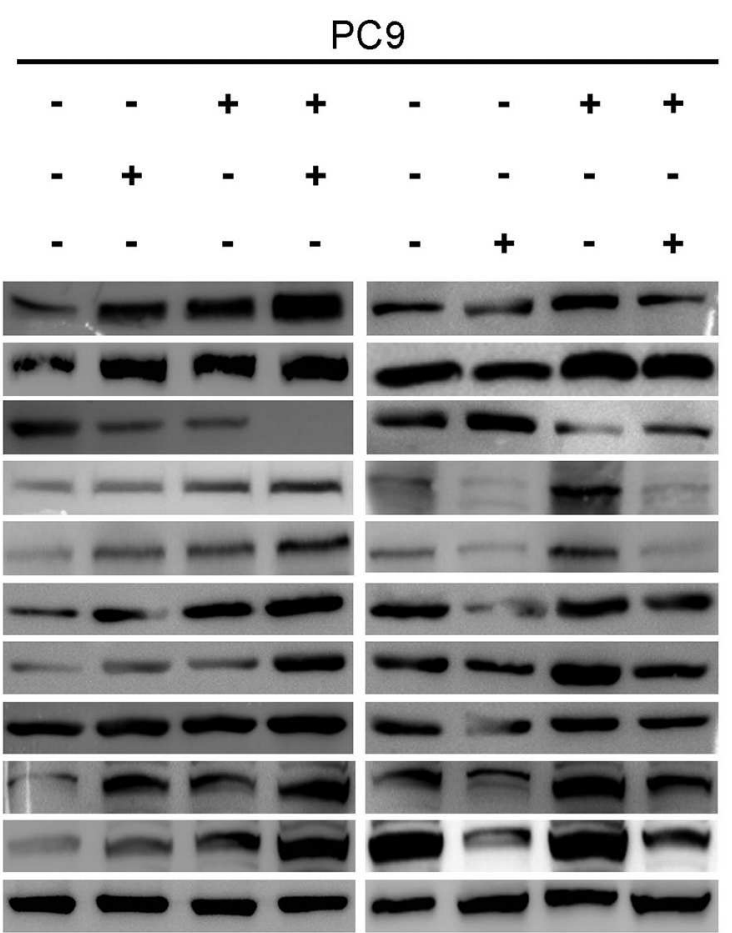

A549

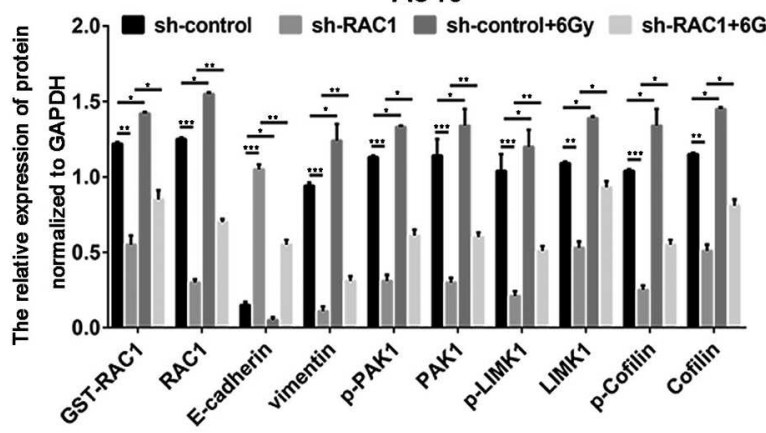

PC9

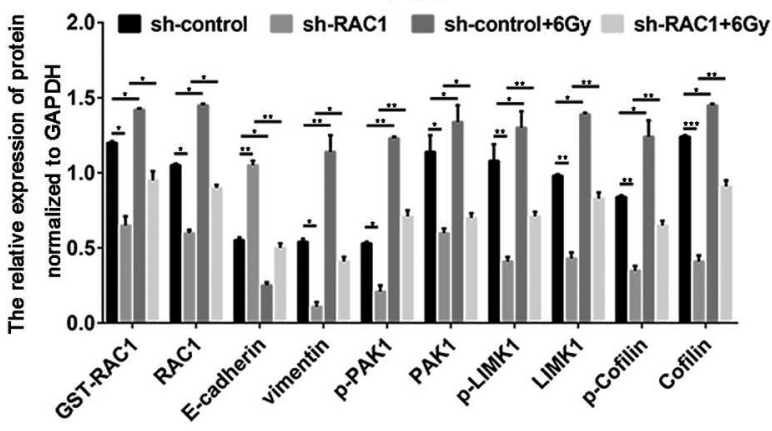

FIGURE 5 | IR induced EMT progression by activating Rac1 signaling pathway in lung cancer cells. A549 and PC9 cells were exposed to 6 Gy radiation in the overexpression and the absence of Rac1 expression. The expression of EMT-related markers, Rac1 expression and activity, and Rac1 signaling pathway related markers (indicated in the figure) was monitored in response to IR treatment. Data are expressed as the mean \pm SD of different groups of cells from three separate experiments. ${ }^{\star} P<0.05,{ }^{\star *} P<0.01,{ }^{* \star} P<0.001$.

The effect of the alterations on radiosensitivity caused by RAC1 inhibition is markedly increased in radiosensitivity of pancreatic cancer cells, as demonstrated by caspase- 3 activation
(32). In this study, we also found that overexpression of Racl significantly promoted the migration and invasion, and radioresistance of lung cancer cells, whereas the knockdown 
A

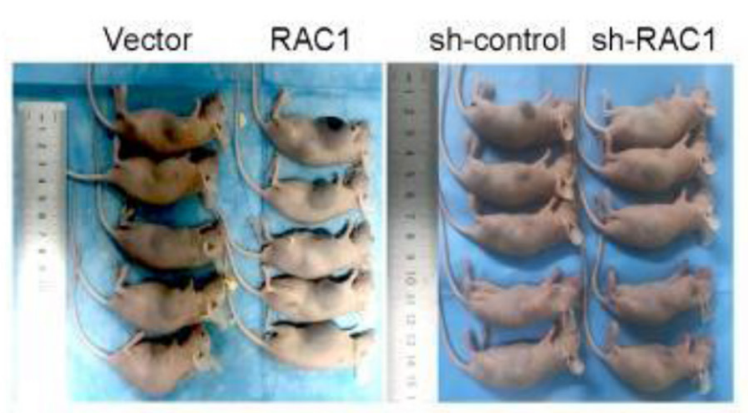

c
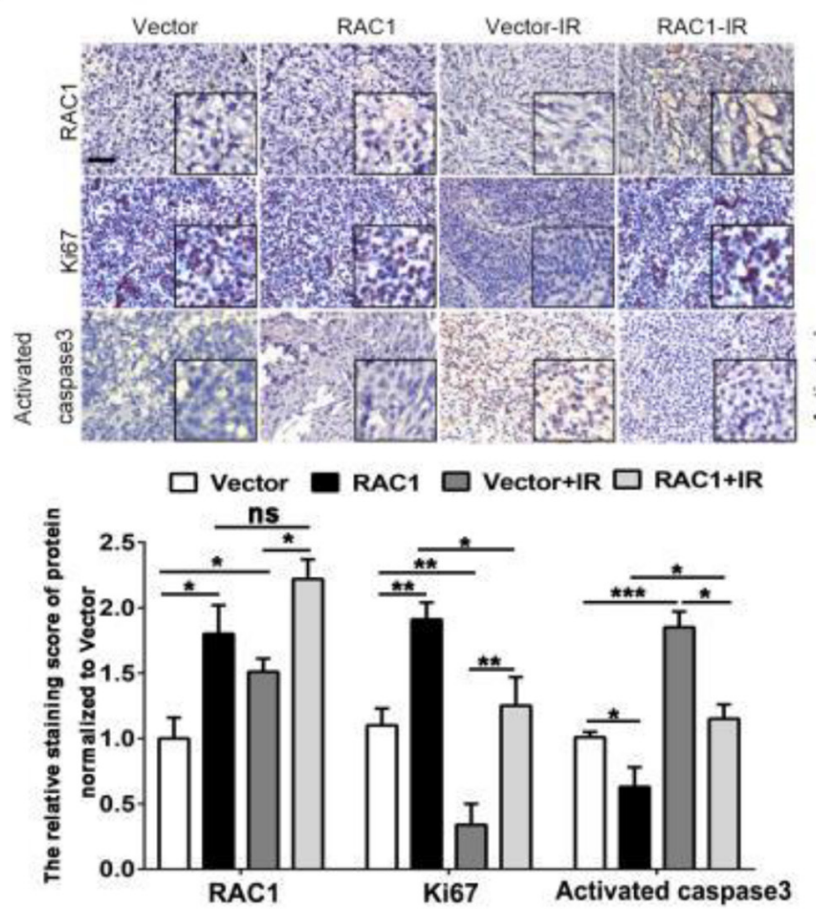

B
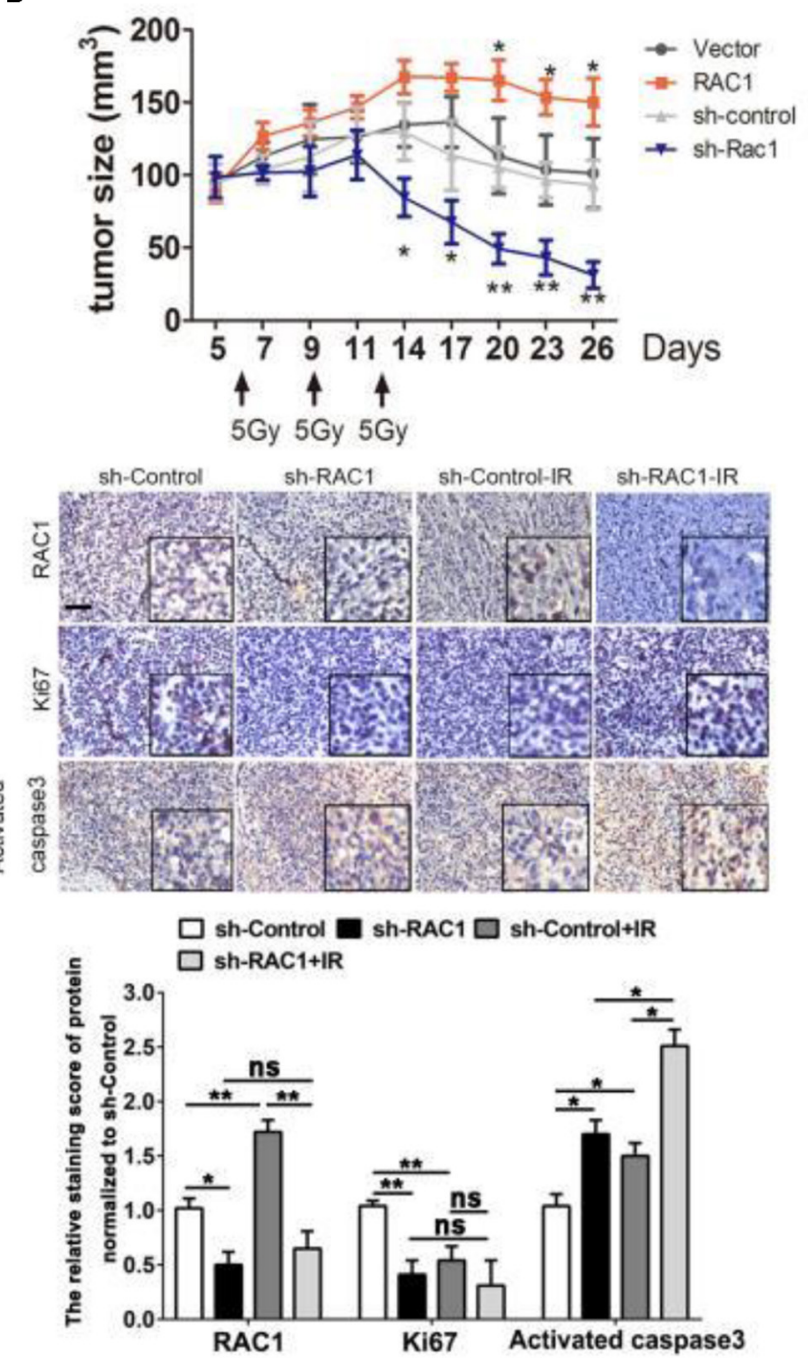

FIGURE 6 | The effects of RAC1 expression in radiotherapy in vivo. (A) Representative photo of residual tumor of after $3{ }^{\star} 5$ Gy dose of irradiation. (B) The time course of growth of Vector, RAC1, sh-control and sh-RAC1 xenograft tumors with or without IR treatment. (C) IHC staining showed an elevated Ki67 expression and upregulation of RAC1 in RAC1 overexpression xenograft tumor, which represents the radioresistant process in RAC1 overexpressing cells in vivo. Right panel showed a decreased Ki67 expression and downregulation of RAC1 in silencing RAC1 xenograft tumor, which represents the radiosensitivity process in sh-RAC1 cells in vivo. Down panel showed the quantification of scoring of immunostaining in RAC1 overexpression/silencing xenograft. Scale bar $100 \mu \mathrm{m}$. Data are expressed as the mean $\pm \mathrm{SD}$ of different groups of cells from three separate experiments. ${ }^{\star} P<0.05,{ }^{\star \star} P<0.01,{ }^{\star \star \star} P<0.001$.

of Rac1 markedly inhibited these capabilities of lung cancer cells in vivo and in vitro. Further studies will be needed to test this possibility and to decipher the mechanisms involved. IR can also simultaneously induce multiple signaling pathways that promote cell survival, such as AKT, ATM/ATR, and ERK cascades (56). It is well-known that the pro-survival signaling pathways generally lead to suppression of apoptosis, activation of cell cycle checkpoint and initiation of DNA repair in cancer cells. By reducing the magnitude of IR-induced cytotoxicity and these pro-survival pathways promote radioresistance in cancer cells. Here, we uncover a novel function for RAC1 signaling in the survival of lung cancer cells in response to IR, suggesting the oncogenetic roles of RAC1 in radioresistance.
Our previous study found that RAC1 could significantly induce the EMT of colon cancer cells, which may be related to the positive regulation of Rac1/PAK1/LIMK1/Cofilins signaling pathway (36). In the present study, we found that IR promoted lung cancer cell EMT in the process, accompanied with an upregulation of RAC1, PAK1, p-PAK1, LIMK1, p-LIMK1, Cofilin, p-Cofilin in these cells. Silencing Racl significantly inhibited the EMT phenotype in lung cancer cells, accompanied with a significant down-regulation of RAC1, PAK1, p-PAK1, LIMK1, pLIMK1, Cofilin, and p-Cofilin in lung cancer cells. Additionally, IR could enhance the expression and activation of RAC1, positively associated with the up-regulation of PAK1, p-PAK1, LIMK1, p-LIMK1, Cofilin and p-Cofilin. Besides, the effect of 
IR on EMT and these molecular markers of Racl signaling pathway could be partly reversed by Racl knockdown, but augmented by Racl overexpression. Thus, a better understanding of the mechanisms that promote survival following IR would potentially allow for the identification of novel therapeutic targets to be explored for radiosensitization of lung cancer cells.

\section{DATA AVAILABILITY STATEMENT}

The datasets generated for this study are available on request to the corresponding author.

\section{ETHICS STATEMENT}

The animal study was reviewed and approved by the Institutional Animal Care and Use Committee of the Hunan Cancer Hospital and The Affiliated Cancer Hospital of Xiangya School of Medicine, Central South University.

\section{AUTHOR CONTRIBUTIONS}

YZ, QL, ST, HeW, PY, and LX designed the experiments and wrote the paper. ST, HeW, PY, LX, LO, JLin, JLia,

\section{REFERENCES}

1. Rudin CM, Poirier JT, Byers LA, Dive C, Dowlati A, George J, et al. Author correction: molecular subtypes of small cell lung cancer: a synthesis of human and mouse model data. Nat Rev Cancer. (2019) 19:415. doi: 10.1038/s41568-019-0164-2

2. Kashima J, Kitadai R, Okuma Y. Molecular and morphological profiling of lung cancer: a foundation for "next-generation" pathologists and oncologists. Cancers (Basel). (2019) 11:E599. doi: 10.3390/cancers11 050599

3. Pirker R. Treatment of advanced non-small-cell lung cancer: from chemotherapy to chemoimmunotherapy. J Oncol Pract. (2018) 14:537-8. doi: $10.1200 / J O P .18 .00474$

4. Okami J. Treatment strategy and decision-making for elderly surgical candidates with early lung cancer. J Thorac Dis. (2019) 11:S987-97. doi: 10.21037/jtd.2019.04.01

5. Pourhanifeh MH, Sharifi M, Reiter RJ, Davoodabadi A, Asemi Z. Melatonin and non-small cell lung cancer: new insights into signaling pathways. Cancer Cell Int. (2019) 19:131. doi: 10.1186/s12935-019-0853-7

6. Bica-Pop C, Cojocneanu-Petric R, Magdo L, Raduly L, Gulei D, BerindanNeagoe I. Overview upon miR-21 in lung cancer: focus on NSCLC. Cell Mol Life Sci. (2018) 75:3539-51. doi: 10.1007/s00018-018-2877-x

7. Park G, Son B, Kang J, Lee S, Jeon J, Kim JH, et al. LDR-induced miR$30 \mathrm{a}$ and miR-30b target the PAI-1 pathway to control adverse effects of NSCLC radiotherapy. Mol Ther. (2019) 27:342-54. doi: 10.1016/j.ymthe.2018. 10.015

8. Kwon OS, Hong SK, Kwon SJ, Go YH, Oh E, Cha HJ. BCL2 induced by LAMTOR3/MAPK is a druggable target of chemoradioresistance in mesenchymal lung cancer. Cancer Lett. (2017) 403:48-58. doi: 10.1016/j.canlet.2017.05.019

9. Wang H, Wang Z, Li Y, Lu T, and Hu G. Silencing snail reverses epithelialmesenchymal transition and increases radiosensitivity in hypopharyngeal carcinoma. Onco Targets Ther. (2020) 13:497-511. doi: 10.2147/OTT.S237410

10. Fu Z, Yu W, Wang H, Chen X. Overexpression of RNF187 induces cell EMT and apoptosis resistance in NSCLC. J Cell Physiol. (2019) 234:14161-9. doi: $10.1002 /$ jcp. 28111
YT, LT, SR, and BZ conducted the experiments. JLin, YH, $\mathrm{HuW}, \mathrm{YS}, \mathrm{QP}, \mathrm{MS}$ and JLia collected the clinical data and sample disposal. All authors read and approved the final manuscript.

\section{FUNDING}

This work was supported in part by grants from the following sources: the National Natural Science Foundation of China (81972636, 81872281), the Natural Science Foundation of Hunan Province (2019JJ40175, 2018JJ1013, 2017JJ2010, 2017JJ3190), the Research Project of the Health and Family Planning Commission of Hunan Province (B20180400, B20180582), the Changsha Science and Technology Board (kq1706045, kq1706043), and Ascend Foundation of National Cancer Center (NCC2018b68).

\section{SUPPLEMENTARY MATERIAL}

The Supplementary Material for this article can be found online at: https://www.frontiersin.org/articles/10.3389/fonc. 2020.00649/full\#supplementary-material

11. Zhou J, Zhu X, Wu S, Guo J, Zhang K, Xu C, et al. Epithelialmesenchymal transition status of circulating tumor cells in breast cancer and its clinical relevance. Cancer Biol Med. (2020) 17:169-80. doi: 10.20892/j.issn.2095-3941.2019.0118

12. Hein AL, Ouellette MM, Yan Y. Radiation-induced signaling pathways that promote cancer cell survival. Int J Oncol. (2014) 45:1813-9. doi: 10.3892/ijo.2014.2614

13. Han D, Wu G, Chang C, Zhu F, Xiao Y, Li Q, et al. Disulfiram inhibits TGF-beta-induced epithelial-mesenchymal transition and stem-like features in breast cancer via ERK/NF-kappaB/Snail pathway. Oncotarget. (2015) 6:40907-19. doi: 10.18632/oncotarget.5723

14. Ungefroren $\mathrm{H}$, Witte D, Lehnert $\mathrm{H}$. The role of small GTPases of the Rho/Rac family in TGF-beta-induced EMT and cell motility in cancer. Dev Dyn. (2018) 247:451-61. doi: 10.1002/dvdy.24505

15. Lv WL, Liu Q, An JH, Song XY. Scutellarin inhibits hypoxia-induced epithelial-mesenchymal transition in bladder cancer cells. J Cell Physiol. (2019) 234:23169-75. doi: 10.1002/jcp.28883

16. Marchand B, Pitarresi JR, Reichert M, Suzuki K, Laczko D, Rustgi AK. PRRX1 isoforms cooperate with FOXM1 to regulate the DNA damage response in pancreatic cancer cells. Oncogene. (2019) 38:4325-39. doi: 10.1038/s41388-019-0725-6

17. Principe DR, Diaz AM, Torres C, Mangan RJ, DeCant B, McKinney $\mathrm{R}$, et al. TGFbeta engages MEK/ERK to differentially regulate benign and malignant pancreas cell function. Oncogene. (2017) 36:4336-48. doi: 10.1038/onc.2016.500

18. Wang Q, Ma J, Lu Y, Zhang S, Huang J, Chen J, et al. CDK20 interacts with KEAP1 to activate NRF2 and promotes radiochemoresistance in lung cancer cells. Oncogene. (2017) 36:5321-30. doi: 10.1038/onc.2017.161

19. Wang Y, Gudikote J, Giri U, Yan J, Deng W, Ye R, et al. RAD50 expression is associated with poor clinical outcomes after radiotherapy for resected non-small cell lung cancer. Clin Cancer Res. (2018) 24:341-50. doi: 10.1158/1078-0432.CCR-17-1455

20. Piscitello D, Varshney D, Lilla S, Vizioli MG, Reid C, Gorbunova $\mathrm{V}$, et al. AKT overactivation can suppress DNA repair via p70S6 kinase-dependent downregulation of MRE11. Oncogene. (2018) 37:427-38. doi: 10.1038/onc.2017.340 
21. Borisova ME, Voigt A, Tollenaere MAX, Sahu SK, Juretschke T, Kreim N, et al. p38-MK2 signaling axis regulates RNA metabolism after UV-light-induced DNA damage. Nat Commun. (2018) 9:1017. doi: 10.1038/s41467-018-03417-3

22. Sertic, S., Mollica, A., Campus, I., Roma, S., Tumini, E., Aguilera, A., et al. (2018). Coordinated activity of Y family TLS polymerases and EXO1 protects non-S phase cells from UV-induced cytotoxic lesions. Mol Cell. 70, 34-47 e34. doi: 10.1016/j.molcel.2018.02.017

23. Alexandru O, Sevastre AS, Castro J, Artene SA, Tache DE, Purcaru OS, et al. Platelet-derived growth factor receptor and ionizing radiation in high grade glioma cell lines. Int J Mol Sci. (2019) 20:4663. doi: 10.3390/ijms20194663

24. Zeng Q, Liu YM, Liu J, Han J, Guo JX, Lu S, et al. Inhibition of ZIP4 reverses epithelial-to-mesenchymal transition and enhances the radiosensitivity in human nasopharyngeal carcinoma cells. Cell Death Dis. (2019) 10:588. doi: 10.1038/s41419-019-1807-7

25. Jung JW, Hwang SY, Hwang JS, Oh ES, Park S, Han IO. Ionising radiation induces changes associated with epithelial-mesenchymal transdifferentiation and increased cell motility of A549 lung epithelial cells. Eur J Cancer. (2007) 43:1214-24. doi: 10.1016/j.ejca.2007.01.034

26. Theys J, Jutten B, Habets R, Paesmans K, Groot AJ, Lambin P, et al. E-Cadherin loss associated with EMT promotes radioresistance in human tumor cells. Radiother Oncol. (2011) 99:392-7. doi: 10.1016/j.radonc.2011.05.044

27. Zhou YC, Liu JY, Li J, Zhang J, Xu YQ, Zhang HW, et al. Ionizing radiation promotes migration and invasion of cancer cells through transforming growth factor-beta-mediated epithelial-mesenchymal transition. Int J Radiat Oncol Biol Phys. (2011) 81:1530-7. doi: 10.1016/j.ijrobp.2011.06.1956

28. Yasser M, Shaikh R, Chilakapati MK, Teni T. Raman spectroscopic study of radioresistant oral cancer sublines established by fractionated ionizing radiation. PLoS ONE. (2014) 9:e97777. doi: 10.1371/journal.pone.0097777

29. Bosco EE, Mulloy JC, Zheng Y. Rac1 GTPase: a "Rac" of all trades. Cell Mol Life Sci. (2009) 66:370-4. doi: 10.1007/s00018-008-8552-x

30. Leng R, Liao G, Wang H, Kuang J, Tang L. Rac1 expression in epithelial ovarian cancer: effect on cell EMT and clinical outcome. Med Oncol. (2015) 32:329. doi: 10.1007/s12032-014-0329-5

31. Nie F, Wang XF, Zhao SY, Bu L, Liu XH. Gene silencing of Rac1 with RNA interference mediated by ultrasound and microbubbles in human LoVo cells: evaluation of cell invasion inhibition and metastatic. J Drug Target. (2015) 23:380-6. doi: 10.3109/1061186X.2014.1002500

32. Hein AL, Post CM, Sheinin YM, Lakshmanan I, Natarajan A, Enke CA, et al. RAC1 GTPase promotes the survival of breast cancer cells in response to hyper-fractionated radiation treatment. Oncogene. (2016) 35:6319-29. doi: 10.1038/onc.2016.163

33. Yan Y, Hein AL, Etekpo A, Burchett KM, Lin C, Enke CA, et al. Inhibition of RAC1 GTPase sensitizes pancreatic cancer cells to gamma-irradiation. Oncotarget. (2014) 5:10251-70. doi: 10.18632/oncotarget.2500

34. Wang H, Zhou Y, Oyang L, Han Y, Xia L, Lin J, et al. LPLUNC1 stabilises PHB1 by counteracting TRIM21-mediated ubiquitination to inhibit NFkappaB activity in nasopharyngeal carcinoma. Oncogene. (2019) 38:5062-75. doi: 10.1038/s41388-019-0778-6

35. Yang $\mathrm{Y}$, Liao Q, Wei F, Li X, Zhang $\mathrm{W}$, Fan S, et al. LPLUNC1 inhibits nasopharyngeal carcinoma cell growth via down-regulation of the MAP kinase and cyclin D1/E2F pathways. PLoS ONE. (2013) 8:e62869. doi: 10.1371/journal.pone.0062869

36. Xia L, Lin J, Su J, Oyang L, Wang H, Tan S, et al. Diallyl disulfide inhibits colon cancer metastasis by suppressing Rac1-mediated epithelial-mesenchymal transition. Onco Targets Ther. (2019) 12:5713-28. doi: 10.2147/OTT.S208738

37. Wei F, Tang L, He Y, Wu Y, Shi L, Xiong F, et al. BPIFB1 (LPLUNC1) inhibits radioresistance in nasopharyngeal carcinoma by inhibiting VTN expression. Cell Death Dis. (2018) 9:432. doi: 10.1038/s41419-018-0409-0

38. Zhou Y, Liao Q, Han Y, Chen J, Liu Z, Ling H, et al. Rac1 overexpression is correlated with epithelial mesenchymal transition and predicts poor prognosis in non-small cell lung cancer. J Cancer. (2016) 7:2100-9. doi: $10.7150 /$ jca. 16198

39. De P, Aske JC, Dey N. RAC1 takes the lead in solid tumors. Cells. (2019) 8:E382. doi: 10.3390/cells8050382

40. Danopoulos S, Krainock M, Toubat O, Thornton M, Grubbs B, Al Alam D. Racl modulates mammalian lung branching morphogenesis in part through canonical Wnt signaling. Am J Physiol Lung Cell Mol Physiol. (2016) 311:L1036-49. doi: 10.1152/ajplung.00274.2016
41. Winge MCG, Marinkovich MP. Epidermal activation of the small GTPase Rac1 in psoriasis pathogenesis. Small GTPases. (2019) 10:163-8. doi: 10.1080/21541248.2016.1273861

42. Poudel KR, Roh-Johnson M, Su A, Ho T, Mathsyaraja H, Anderson S, et al. Competition between TIAM1 and membranes balances endophilin A3 activity in cancer metastasis. Dev Cell. (2018) 45:738-52 e736. doi: 10.1016/j.devcel.2018.05.021

43. Zou Y, Xu S, Xiao Y, Qiu Q, Shi M, Wang J, et al. Long noncoding RNA LERFS negatively regulates rheumatoid synovial aggression and proliferation. J Clin Invest. (2018) 128:4510-24. doi: 10.1172/JCI97965

44. Chelvanambi S, Gupta SK, Chen X, Ellis BW, Maier BF, Colbert TM, et al. HIV-Nef protein transfer to endothelial cells requires Racl activation and leads to endothelial dysfunction implications for statin treatment in HIV patients. Circ Res. (2019) 125:805-20. doi: 10.1161/CIRCRESAHA.119.315082

45. Goka ET, Chaturvedi P, Lopez DTM, Garza A, Lippman ME. RAC1b overexpression confers resistance to chemotherapy treatment in colorectal cancer. Mol Cancer Ther. (2019) 18:957-68. doi: 10.1158/1535-7163.MCT-18-0955

46. Maskell D, Geropantas K, Kouroupis M, Glenn A, Ajithkumar T. Treatment of choice for patients with EGFR mutation-positive non-small cell lung carcinoma presenting with choroidal metastases: radiotherapy or TKIs? Can J Ophthalmol. (2017) 52:e22-5. doi: 10.1016/j.jcjo.2016.09.010

47. Sun J, Liu NB, Zhuang HQ, Zhao LJ, Yuan ZY, Wang P. Celecoxib-erlotinib combination treatment enhances radiosensitivity in A549 human lung cancer cell. Cancer Biomark. (2017) 19:45-50. doi: 10.3233/CBM-160323

48. Cui YH, Suh Y, Lee HJ, Yoo KC, Uddin N, Jeong YJ, et al. Radiation promotes invasiveness of non-small-cell lung cancer cells through granulocyte-colonystimulating factor. Oncogene. (2015) 34:5372-82. doi: 10.1038/onc.2014.466

49. Bahrami A, Majeed M, Sahebkar A. Curcumin: a potent agent to reverse epithelial-to-mesenchymal transition. Cell Oncol (Dordr). (2019) 42:405-21. doi: 10.1007/s13402-019-00442-2

50. Jung $\mathrm{CH}$, Han AR, Chung HJ, Ha IH, Um HD. Linarin inhibits radiationinduced cancer invasion by downregulating MMP-9 expression via the suppression of NF-kappaB activation in human non-small-cell lung cancer A549. Nat Prod Res. (2019) 33:3582-6. doi: 10.1080/14786419.2018.1484460

51. Kim JY, Jeon S, Yoo YJ, Jin H, Won HY, Yoon K, et al. The Hsp27-mediated IkBalpha-NFkappaB signaling axis promotes radiation-induced lung fibrosis. Clin Cancer Res. (2019) 25:5364-75. doi: 10.1158/1078-0432.CCR-18-3900

52. Ma H, Li T, Tao Z, Hai L, Tong L, Yi L, et al. NKCC1 promotes EMT-like process in GBM via RhoA and Rac1 signaling pathways. J Cell Physiol. (2019) 234:1630-42. doi: 10.1002/jcp.27033

53. Yan Y, Hein AL, Greer PM, Wang Z, Kolb RH, Batra SK, et al. A novel function of HER2/Neu in the activation of G2/M checkpoint in response to gamma-irradiation. Oncogene. (2015) 34:2215-26. doi: 10.1038/onc.2014.167

54. Wong BS, Shea DJ, Mistriotis P, Tuntithavornwat S, Law RA, Bieber JM, et al. A direct podocalyxin-dynamin-2 interaction regulates cytoskeletal dynamics to promote migration and metastasis in pancreatic cancer cells. Cancer Res. (2019) 79:2878-91. doi: 10.1158/0008-5472.CAN-18-3369

55. Zhou Y, Wang Y, Zhou W, Chen T, Wu Q, Chutturghoon VK, et al. YAP promotes multi-drug resistance and inhibits autophagy-related cell death in hepatocellular carcinoma via the RAC1-ROS-mTOR pathway. Cancer Cell Int. (2019) 19:179. doi: 10.1186/s12935-019-0898-7

56. Chen L, Xia Y, Lu J, Xie Q, Ye A, Sun W. A 50-Hz magnetic-field exposure promotes human amniotic cells proliferation via SphK-S1P-S1PR cascade mediated ERK signaling pathway. Ecotoxicol Environ Saf. (2020) 194:110407. doi: 10.1016/j.ecoenv.2020.110407

Conflict of Interest: The authors declare that the research was conducted in the absence of any commercial or financial relationships that could be construed as a potential conflict of interest.

Copyright $\odot 2020$ Tan, Yi, Wang, Xia, Han, Wang, Zeng, Tang, Pan, Tian, Rao, Oyang, Liang, Lin, Su, Shi, Liao and Zhou. This is an open-access article distributed under the terms of the Creative Commons Attribution License (CC BY). The use, distribution or reproduction in other forums is permitted, provided the original author(s) and the copyright owner(s) are credited and that the original publication in this journal is cited, in accordance with accepted academic practice. No use, distribution or reproduction is permitted which does not comply with these terms. 